\title{
EUV line intensity distribution in the solar atmosphere: differences between a polar coronal hole and its equatorial extension
}

\author{
K. P. Raju^ and B. J. I. Bromage
}

Centre for Astrophysics, University of Central Lancashire, Preston, UK
e-mail: kpr@iiap.res.in, bjibromage@uclan.ac.uk

Received 8 February 2005 / Accepted 14 September 2005

\section{ABSTRACT}

Intensity histograms of 14 EUV emission lines in a polar coronal hole, its equatorial extension, the "Elephant's Trunk", and the adjacent quiet Sun regions have been obtained from SOHO/CDS observations made in August and September 1996. Differences between the quiet region and coronal hole on the one hand, and the polar hole and the equatorial extension on the other, have been examined. As reported earlier, intensity histograms are more similar in the quiet Sun region and the hole for lower transition region lines, while they become increasingly different for upper transition region lines and the corona. However, we also find that coronal holes affect line intensities at most of the atmospheric heights from the transition region to the corona. Histograms of the polar hole and its equatorial extension show dramatic differences with respect to height in the solar atmosphere. The polar coronal hole appears to be brighter than the equatorial extension for emission lines with formation temperature below $0.52 \mathrm{MK}$, while it appears to be increasingly darker above this temperature. Possible reasons for the polarequatorial differences in coronal holes are briefly discussed on the basis of flux transport in the solar atmosphere and the consequences of the different orientations of the observed boundary regions of the two coronal holes. The different behaviour seen in the polar coronal hole and its equatorial extension are explained in terms of the effects resulting from different levels of magnetic reconnection occurring in the two regions under study.

Key words. Sun: corona - Sun: transition region

\section{Introduction}

Coronal holes are the source regions of the fast solar wind that may cause intense geomagnetic storms (Krieger et al. 1973). The acceleration of the fast solar wind has not been understood well and it remains as a fundamental problem in astrophysics. The temperature of the coronal hole is less than a million $\mathrm{K}$ which cannot provide the additional momentum required by the fast solar wind. The velocity of the fast solar wind is $800 \mathrm{~km} \mathrm{~s}^{-1}$ and temperatures of 3-4 MK are required near the base of the corona, according to the Parker model of a thermally driven solar wind (Parker 1958). Other processes suggested for the acceleration mechanism include direct transfer of momentum from MHD waves (David et al. 1998). Spectroscopic observations can provide valuable clues to this problem. SOHO has given us an unprecedented opportunity to make simultaneous spectroscopic observations of the solar atmosphere at different heights. The presence of MHD waves has been reported recently both in the coronal hole and outside, though their detailed properties are yet to be known (Marsh et al. 2002).

^ On leave from, Indian Institute of Astrophysics, Bangalore560034, India.
This paper examines the spatial variations of emission line intensities obtained from SOHO/CDS observations of a Polar Coronal Hole $(\mathrm{PCH})$ and the Equatorial extension of the polar Coronal Hole (ECH), known as the "Elephant's Trunk". Fourteen strong emission lines available in the CDS/NIS wavelength range have been selected to study the variations throughout the solar atmosphere from chromosphere to corona. Differences between the quiet region and coronal hole on the one hand, and the polar hole and the equatorial extension on the other, are examined. The observations were made close to the solar minimum periods when coronal holes have their maximum coverage.

\section{Data}

The observations were part of a programme to study the evolution of coronal holes throughout the solar cycle. The present study uses SOHO/CDS observations made during the Whole Sun Month (August-September 1996). Details of the observations may be seen in Del Zanna (1999), Bromage et al. (2000). The initial data reduction and analysis have been described in Raju et al. (2005) (Paper I). Fourteen of the strong lines were selected from those available in the CDS study which was used. 

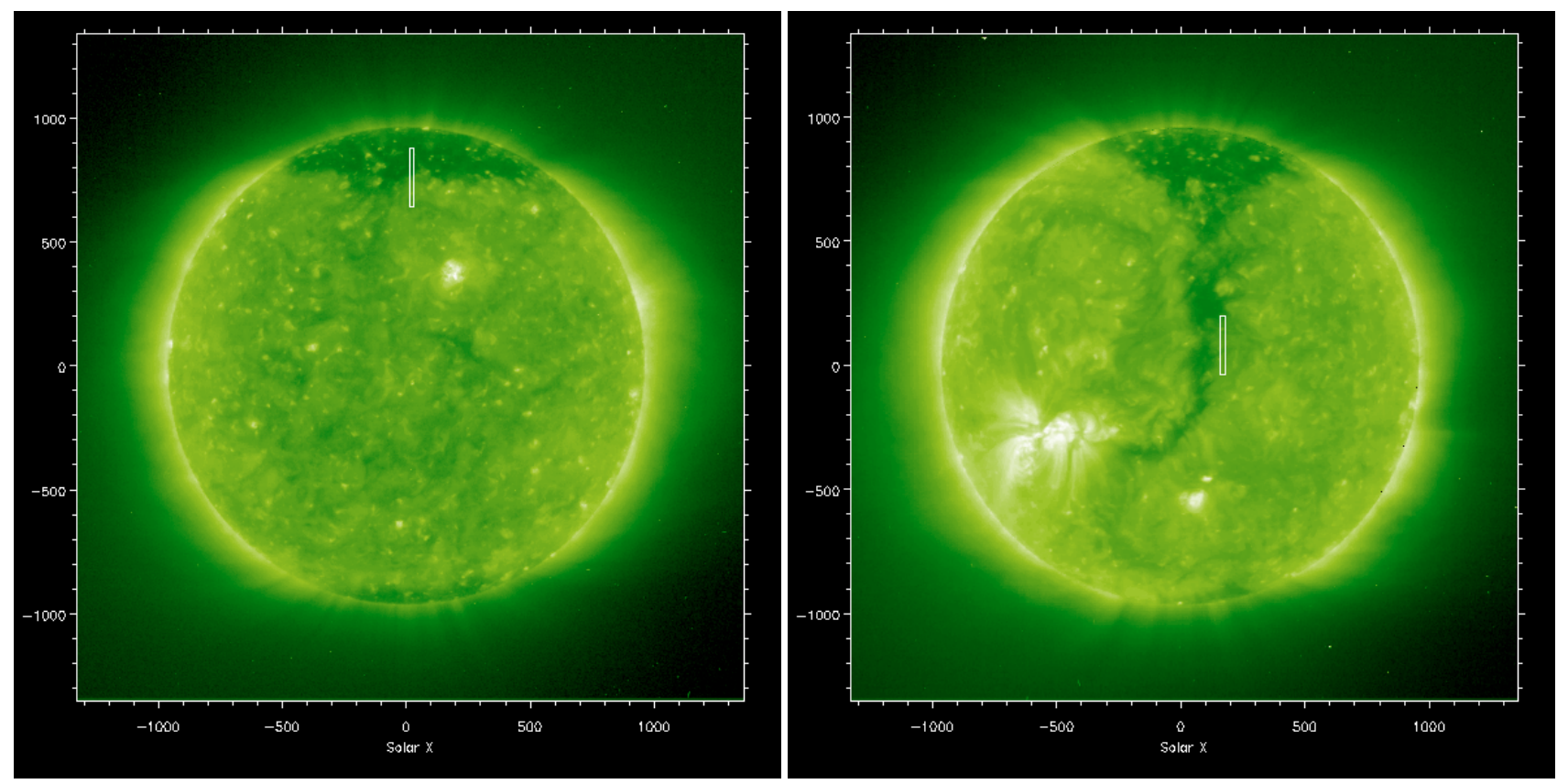

Fig. 1. EIT $195 \AA$ A image and the CDS raster position in the observations of the polar region (left) and the equatorial region (right).

Table 1. Details of emission line parameters.

\begin{tabular}{llll}
\hline \hline No. & Ion & $\begin{array}{l}\lambda \\
{[\AA]}\end{array}$ & $\begin{array}{l}T \\
{[\mathrm{MK}]}\end{array}$ \\
\hline 1 & HeII(2) & 607.56 & 0.083 \\
2 & OIII & 599.59 & 0.11 \\
3 & OIV & 608.40 & 0.19 \\
4 & NeIV & 542.89 & 0.19 \\
5 & OV & 629.73 & 0.25 \\
6 & NeVI & 562.80 & 0.43 \\
7 & NeVII & 561.73 & 0.52 \\
8 & CaX & 557.76 & 0.66 \\
9 & MgVII & 367.7 & 0.66 \\
10 & SiVIII & 316.22 & 0.81 \\
11 & MgVIII & 315.01 & 0.81 \\
12 & MgIX & 368.06 & 0.95 \\
13 & SiIX & 349.9 & 1.07 \\
14 & MgX & 624.94 & 1.10 \\
\hline
\end{tabular}

The temperature of formation of these lines is in the range 0.083-1.10 MK; details may be seen in Table 1. For each observation, the field of view encompasses part of the coronal hole, the hole boundary and the adjacent quiet region. The whole area is a combination of three rasters, resulting in a $240^{\prime \prime} \times 60^{\prime \prime}$ field of view. The spatial resolution is $4^{\prime \prime}$ but the averaging of nearby pixels has reduced this to about $20^{\prime \prime}$ and the overall temporal resolution to about an hour. Altogether there are 21 datasets from 18 days of observation, out of which 14 are from the polar regions near the central meridian, and seven are from the ECH. Examples of typical rasters are shown in Fig. 1.

\section{Results}

The coronal hole and the quiet region were identified in each of the observations from the intensity of the $\mathrm{MgX} 625 \AA$ line.
Two intensity contour levels which represent the coronal hole and the quiet Sun region were found by a trial and error method and by comparing them with the EIT images. The details may be seen in Paper I. The intensity histograms of the fourteen emission lines from the quiet Sun region and the coronal hole were produced, combining all the data sets for each region. These are given in Fig. 2. Note that intensities are the total intensity of the line. The emission line parameters and its formation temperature are given at the top of each plot. An examination of the figure reveals the following: barring He II $304 \AA$ and Ca X $557.7 \AA$, there is a systematic behaviour with respect to the temperature of the solar atmosphere. Intensity histograms of the quiet Sun and the hole are more similar for lower transition region (TR) lines, while they are increasingly different for upper TR lines and the corona. The He line and the Ca line behave as if their line formation temperatures are shifted towards higher temperatures. It may also be seen that histograms of the hole region tend to be higher and narrower as one approaches the corona. Notice also that the lower TR lines OIII $600 \AA$ and OIV $608 \AA$ have histograms which indicate that the coronal holes are slightly brighter than the quiet Sun in polar regions. This is consistent with the result found in Paper I.

An examination of the quiet Sun histograms from the polar and equatorial region reveal no appreciable differences, though a slight brightening of the polar region can be seen sometimes, especially in the coronal lines. However, coronal hole histograms from the polar and equatorial region show dramatic differences. It can be seen that histograms of polar hole for the lower TR lines OIII, OIV, NeIV, OV and NeVI are shifted towards the bright side. At the NeVII level, histograms of both regions nearly coincide. From $\mathrm{Ca} \mathrm{X}$ level onwards, the trend is reversed, i.e., histograms of polar regions show a shift towards the darker side. The anomalous behaviour of the He line can also be seen (see discussion, below). 

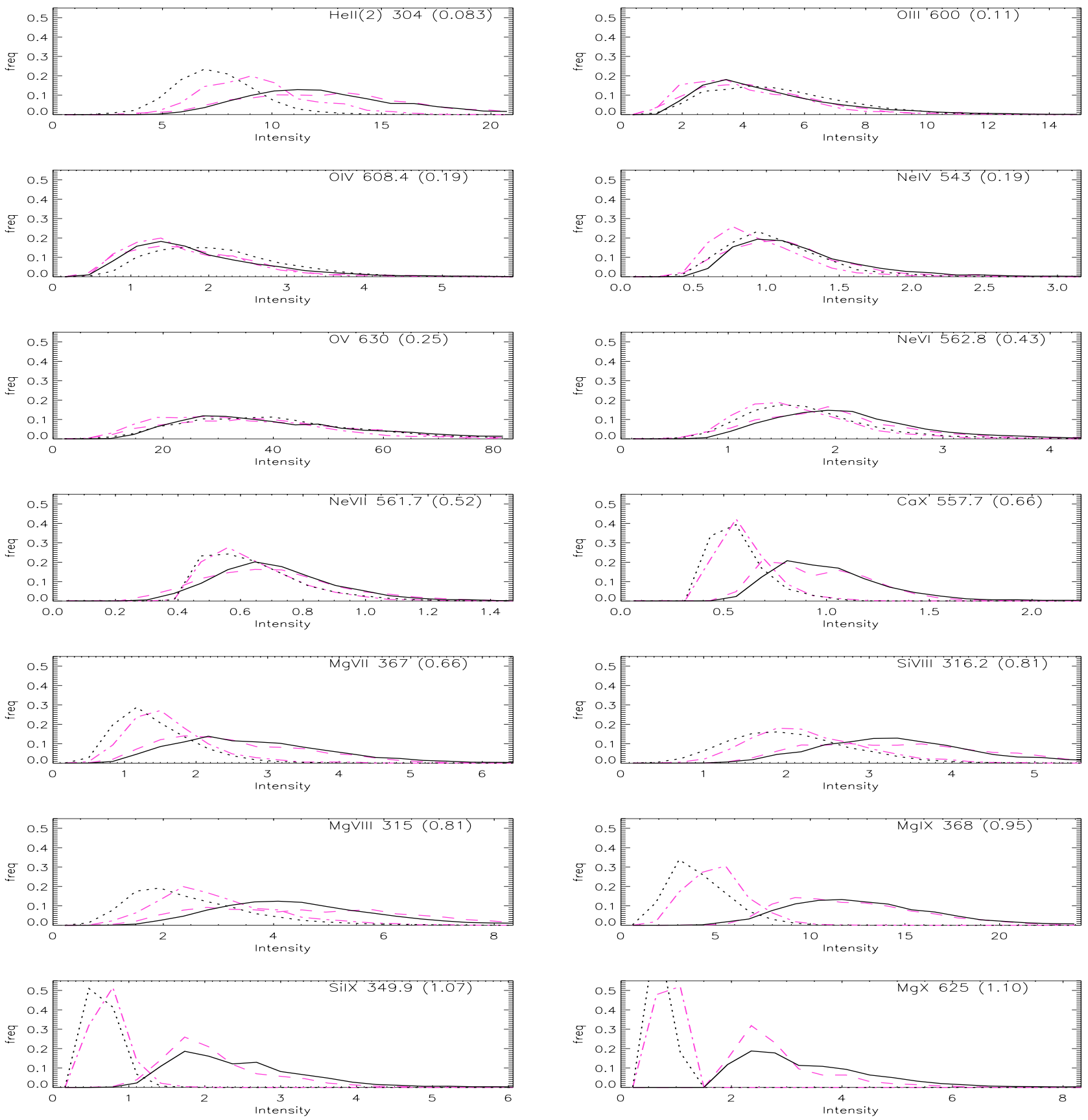

Fig. 2. Intensity histograms of quiet Sun region and coronal hole at various heights in the solar atmosphere. Intensity units are $\left[10^{-3} \mathrm{~W} \mathrm{~m}^{-2} \mathrm{sr}^{-1}\right]$. The emission line parameters and its formation temperature are given at the top of each plot. Solid line represents quiet Sun in polar regions (PQS), dashed line represents quiet Sun in equatorial regions (EQS), dotted line represents coronal hole in polar regions (PCH), and dash-dot line represents coronal hole in equatorial regions $(\mathrm{ECH})$.

From the above distributions, we can get an estimate of how coronal holes affect emission line intensities at various heights of the solar atmosphere. As it is clear from Fig. 2 that the coronal hole behaves differently in the polar and equatorial extension, this is examined separately for the two regions. For this purpose, the ratio of the medians of the intensity distributions is taken for each line for the hole and the quiet Sun region. The ratio is plotted against the line formation temperature in Figs. 3a and b. It can be seen, for example, that in the equatorial extension, the TR line OIII with formation temperature of
$0.11 \mathrm{MK}$ is weaker than the quiet Sun by $15 \%$, while the coronal $\mathrm{Mg} \mathrm{X}$ line, with formation temperature of $1.10 \mathrm{MK}$, is $75 \%$ weaker. In comparison, the OIII line is brighter by $8 \%$ and the $\mathrm{Mg} \mathrm{X}$ line is weaker by $85 \%$, in the polar region than in the adjacent quiet Sun.

In Figs. $3 \mathrm{c}$ and d, we give the polar and equatorial differences for similar regions; intensity ratios of the polar and equatorial hole are plotted in Fig. 3c and intensity ratios of the polar and equatorial quiet Sun region given in Fig. 3d. It can be seen that the polar hole is brighter by about $30 \%$ compared 


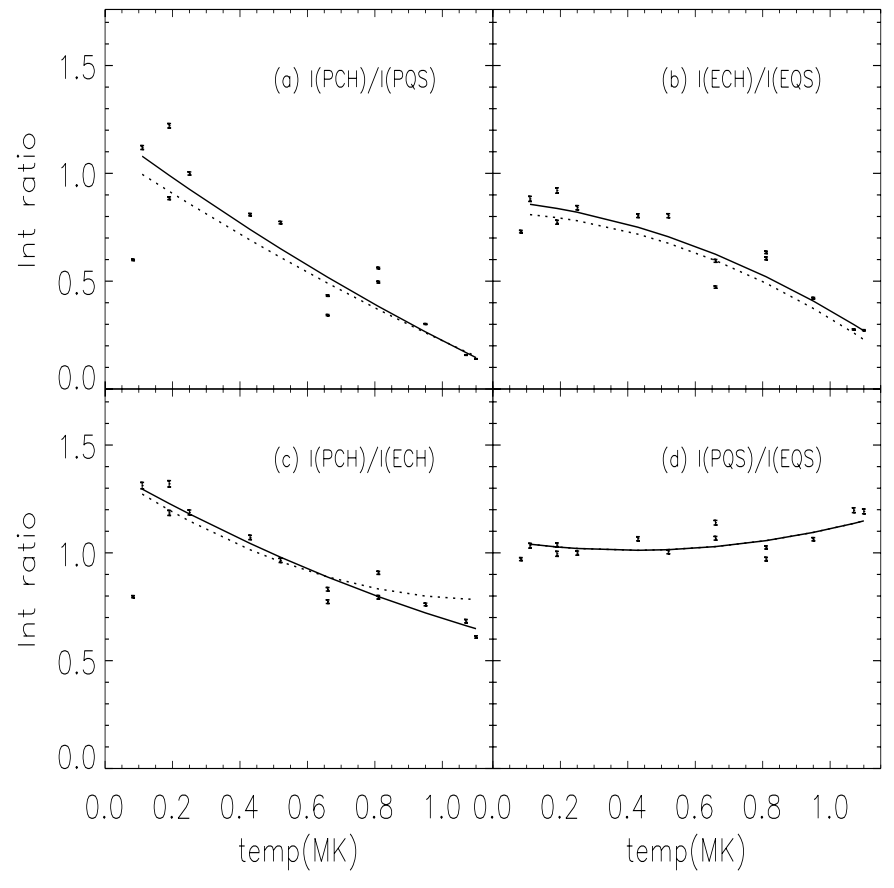

Fig. 3. How a coronal hole influences line intensities at various temperatures in the solar atmosphere is shown separately for polar regions a) and equatorial regions b). Intensity ratios of the polar and equatorial hole are plotted in c) and intensity ratios of the polar and equatorial quiet Sun region are shown in d). A second degree polynomial fit is plotted as a solid line. The dotted line represents an estimate of the effect of a possible EQS contamination of the ECH values.

to its equatorial extension in the lower TR, while it is weaker by about $30 \%$ in the lower corona. Figure $3 \mathrm{~d}$ shows a general brightening of the emission lines in polar regions and a very marginal increase from the lower TR to the corona which is not significant.

\section{Discussion}

The He II $304 \AA$ and Ca X $557.7 \AA$ lines behave as if their formation temperature is shifted upwards. The anomalous behaviour of the He line is well known and is due to the resonance scattering mechanism of line emission and large optical depth (Gallagher et al. 1998; Patsourakos et al. 1999; Jordan et al. 2001). The behaviour of Ca X $557.7 \AA$ line is probably due to the asymmetrical high-temperature wing of its contribution function which is discussed in more detail in Paper I.

Intensity distributions of coronal holes and quiet regions have been described by several authors from the SKYLAB days (Munro \& Withbroe 1972; Huber et al. 1974; Bohlin 1977; Mango et al. 1978; Bocchialini \& Vial 1996; Wilhelm et al. 1998; Stucki et al. 2002). Huber et al. (1974) and Bohlin (1977) point out that compared to the quiet regions outside, intensity histograms of coronal holes are shifted towards the darker side and have a higher and narrower peak indicative of more uniform intensities. Histograms of quiet regions show a pronounced tail towards the brighter side which is due to the network. Hence it is the disappearance of the network that leads to the decrease of intensity in holes. The systematic behaviour seen in our results - intensity histograms of the quiet Sun region and the hole are more similar for lower transition region lines, while they become increasingly different in the upper transition region and the corona - generally agree with this picture.

The most interesting result is the polar-equatorial differences in the intensity distribution of the hole, given in Fig. 2. The $\mathrm{PCH}$ appears to be brighter than the $\mathrm{ECH}$ for lines forming below $T=0.52 \mathrm{MK}$ while the $\mathrm{PCH}$ appears to be darker than the ECH above this temperature. Also, Fig. 3c shows that this change is gradual. The brightness difference in the $\mathrm{PCH}$ and its $\mathrm{ECH}$ with respect to the height in the solar atmosphere is probably reported for the first time. As shown in Fig. 3d, the quiet Sun in the polar region is marginally brighter in all observed lines, but the variation with the height of the solar atmosphere is not significant. Wilhelm et al. (1998) report brightening of the UV lines from lower TR towards the limb which is attributed to spicule absorption. However, they also find that this is true only in cooler lines but is absent in the hotter lines of Ne VIII and $\mathrm{Mg} \mathrm{X}$.

Limb brightening has been observed in polar coronal holes (Munroe \& Withbroe 1972; Huber et al. 1974). A limbbrightened ring is generally reported in the off-limb observations above the polar coronal hole. This has several important consequences for the energy balance of the TR. The thickness of the TR layer is estimated to be five times larger within the coronal hole than in a quiet Sun region and consequently temperature gradients are five times lower in coronal holes. This, in turn, led to the important conclusion that heat flux conducted from the coronal hole to the underlying TR is smaller by the same factor. This implies that the downward conductive flux is too low to balance the radiative losses in the TR and hence mechanical heating must take place (Withbroe 1977; Withbroe $\&$ Noyes 1977). These calculations are based on the early findings that there is no significant difference between line intensities inside and outside the coronal holes, up to temperatures about $0.8 \mathrm{MK}$. Our results do not agree with these findings. We find that coronal holes affect line intensities at most of the atmospheric heights from the TR to the corona. Hence our results may have some consequences for the energy balance of the TR. Further, we find that coronal holes affect line intensities differently in polar and equatorial regions. The quiet Sun does not show significant differences between polar and equatorial regions which would enable us to compare the line intensities in the PCH and ECH in the lower TR (Paper I). We find evidence of brightening of the $\mathrm{PCH}$ in lower transition region lines and darkening of the $\mathrm{PCH}$ in upper TR lines, relative to the ECH. Hence, more importantly, the results point to the different physical conditions existing in the $\mathrm{PCH}$ and its $\mathrm{ECH}$.

There is some lack of consensus on the nature of intensity distributions of the coronal hole and outside in the lower atmosphere. No differences have generally been observed between the intensity histograms of coronal hole and quiet region at chromospheric levels (Bohlin 1977), though Marsh (1977) reports difference in the $\mathrm{Ca} \mathrm{K}$ line. From OSO 8 observations of an equatorial coronal hole in 1975, Bocchialini \& Vial (1996) find that the line profiles in hydrogen Ly $\alpha$ and Mg II are stronger in the hole than outside, while the chromospheric 
Ca II line profiles do not show any difference. Mango et al. (1978) studied centre-to-limb variation of He II $304 \AA$ \& $256 \AA$ and He I $584 \AA$ \& $537 \AA$ and found limb brightening for quiet Sun region, limb neutrality for unipolar magnetic regions and limb darkening in PCH. The behaviour of the He II line in our observations is very similar.

The line of sight effects from the centre of the disk towards the limb, can probably be ruled out because the quiet Sun region adjacent to the hole, though always lying nearer to the centre by a few degrees, does not show any appreciable trend with height. There could also be other causes for the polar-equatorial asymmetry in the coronal hole emission such as spicule absorption or the effects of hot overlying layers. However, these effects are not expected to give such systematic trends. Yet another possibility is due to the proximity of the points in the ECH to the hole boundary. Statistically, points in ECH observations lie closer to the boundary and this can affect the line intensity. The effect can be estimated by avoiding the coronal hole points which lie too close to the boundary and by selecting those points within a fixed distance from the boundary for which the average distance to the boundary is the same in both PCH and ECH. The intensity ratios are re-calculated with these criteria and are plotted in Fig. 3 as dotted lines. It can be seen that the ratios are affected by only a few percent and hence the EQS contamination of ECH can generally be ignored. However, the effect is about $14 \%$ in $\mathrm{I}(\mathrm{PCH}) / \mathrm{I}(\mathrm{ECH})$ (Fig. 3c) at the higher temperature end, which somewhat reduces the significance of ECH brightening.

The flux transport in the solar surface is carried out by supergranulation, meridional circulation, and differential rotation. Supergranular diffusion is expected to be isotropic and hence can not contribute to the differences between ECH and $\mathrm{PCH}$; the differential rotation moves the flux in longitude and the rotational shearing is maximum in midlatitudes (Wang \& Sheeley 1990), while the meridional circulation carries the flux at the surface poleward. The polar coronal hole boundary has been known to increase in brightness as the cycle rises from minimum in mid-1996 to the early new cycle in 1997. An arcade of quiet Sun, closed field surrounds the boundary with the footpoints nearest the coronal hole having the same polarity as the open field in the coronal hole. As the meridional circulation drives more flux towards the poles, the uni-polar open field within the coronal hole acts as a barrier to poleward motion of the coronal field. As the flux builds up, the field in the boundary becomes compressed, allowing shearing to occur which may lead to magnetic reconnection events taking place between the open and closed magnetic field. In this way, the boundary region becomes hotter and brighter as a result of the meridional flow which is moving perpendicular to the boundary.

In Paper I we showed that observations of enhanced emission in lower transition region lines within coronal holes could be explained by thermal conduction of electrons down the field lines from such reconnection events in the corona.

Meridional circulation would not produce the same effect in the equatorial coronal hole because of the alignment of this hole in a north-south direction. However, an analogous behaviour can occur at the eastern edge of the coronal hole because of the effect of quasi-rigid rotation observed in such features. At latitudes away from the equator, the apparent difference in rotation rates, between the differential rate in the photosphere and the quasi-rigid coronal rotation, causes the coronal plasma to be impeded in its rotation from $\mathrm{E}$ to $\mathrm{W}$, in the same way that the meridional flow of plasma poleward is impeded at the polar hole boundary. This could also lead to an enhancement in magnetic reconnection at the $\mathrm{E}$ boundary of the coronal hole, and this is consistent with the observations reported by Bromage et al. (2000), that the eastern boundary of the coronal hole is hotter than the the western edge. However, the ECH observations presented here were in fact close to the western boundary, not the eastern one; here, the lower temperature implies less reconnection events per unit area (consistent with the observed weaker coronal field on this side of the hole). With less reconnection events, the thermal conduction down the field lines is reduced and the brightness of the coronal hole in the lower TR is reduced towards that of the adjacent quiet Sun (as shown in Paper I).

So, the enhanced coronal brightening at the PCH boundary and the lack of such an enhancement at the western ECH boundary can explain the result shown in the lower temperature regions of Fig. 3c. In coronal lines, the ECH appears brighter than the $\mathrm{PCH}$, but as seen above, the EQS contamination of the ECH has a significant contribution here. Hence this brightening needs to be further verified through future studies.

\section{Conclusions}

The intensity distributions of 14 strong EUV emission lines with a temperature of line formation in the range $0.083 \mathrm{MK}$ to $1.10 \mathrm{MK}$ in a PCH, ECH and the adjacent quiet Sun region have been obtained. As is known from SKYLAB days, we see a systematic behaviour in the histograms; intensity histograms of the quiet Sun region and the hole are more similar for lower transition region lines, while they become increasingly different, when passing from the upper transition region into the corona. However, we also find that coronal holes affect line intensities at most of the atmospheric heights through the TR to the corona. It has been found that emission lines are affected quite differently in the $\mathrm{PCH}$ and the ECH. For example, in the ECH, the TR line OIII with formation temperature of $0.11 \mathrm{MK}$ is weaker than the adjacent quiet Sun by $15 \%$, while the coronal $\mathrm{Mg} \mathrm{X}$ line, with formation temperature of $1.10 \mathrm{MK}$, is $75 \%$ weaker. In comparison, the OIII line is brighter by $8 \%$ and the $\mathrm{Mg} \mathrm{X}$ line is weaker by $85 \%$, in the $\mathrm{PCH}$ than the nearby quiet Sun region.

The quiet Sun region near the pole shows a marginal brightening compared to the equatorial regions in all the observed lines, though no significant height variation is observed. However, the coronal holes show polar-equatorial differences in the intensity distribution with respect to the height in the solar atmosphere. The PCH appears to be brighter than the ECH for lines forming below $T=0.52 \mathrm{MK}$, while the PCH increasingly appears to be darker than the ECH above this temperature. The polar hole is brighter by about $30 \%$ compared to its equatorial extension in the lower TR, while it is weaker by about $30 \%$ in the lower corona. Possible reasons for the polarequatorial differences in coronal holes are explained on the 
basis of the different orientation of the boundaries of the two holes, and the consequent effects of different levels of magnetic reconnection which may be occurring in the boundary regions of the two types of coronal hole.

It is also found that the intensity histograms of He II $304 \AA$ and Ca X $557.7 \AA$ lines do not conform to the general picture but behave as if their formation temperature is higher than expected.

Acknowledgements. K.P. Raju is supported by a PPARC research grant. Data are provided courtesy of SOHO/CDS consortium. SOHO is a project of international cooperation between ESA and NASA. The authors are grateful to the CDS project team and the EIT consortium for their support.

\section{References}

Bocchialini, K., \& Vial, J.-C. 1996, Sol. Phys., 168, 37

Bohlin, J. D. 1977, in Coronal Holes and High Speed Wind Streams, ed. J. B. Zirker (Boulder, Col.: Colorado Associated Press)

Bromage, B. J. J., Alexander, D., Breen, A., et al. 2000, Sol. Phys., 193,181

David, C., Gabriel, A. H., Bely-Dubau, F., et al. 1998, A\&A, 336, L90

Del Zanna, G. 1999, Ph.D. Thesis, Univ. of Central Lancashire
Gallagher, P. T., Phillips, K. J. H., Harra-Murnion, L. K., \& Keenan, F. P. 1999, A\&A, 335, 733G

Huber, M. C. E., Foukal, P. V., Noyes, R. W., et al. 1974, ApJ, 194, L115

Jordan, C., Macpherson, K. P., \& Smith, G. R. 2001, MNRAS, 328, $1098 \mathrm{~J}$

Krieger, A. S., Timothy, A. F., \& Roelof, E. C. 1973, Sol. Phys., 29, 505

Mango, S. A., Bohlin, J. D., Glackin, D. L., \& Linsky, J. L. 1978, ApJ, 220,683

Marsh, K. A. 1977, Sol. Phys., 52, 343

Marsh, M. S., Walsh, R. W., \& Bromage, B. J. I. 2002, A\&A, 393, 649

Munro, R. H., \& Withbroe, G. L. 1972, ApJ, 176, 511

Parker, E. N. 1958, ApJ, 128, 664

Patsourakos, S., Vial, J.-C., Gabriel, A. H., \& Bellamine, N. 1999, ApJ, 522, 540

Raju, K. P., Bromage, B. J. I., Chapman, S. A., \& Del Zanna, G. 2005, A\&A, 432, 341

Stucki, K., Solanki, S. K., Pike, C. D., et al. 2002, A\&A, 381, 653

Wang, Y.-M., \& Sheeley, N. R., Jr., D. 1990, ApJ, 365, 372

Wilhelm, K., Lemaire, P., Dammasch, I. E., et al. 1998, A\&A, 334, 685

Withbroe, G. L. 1977, in Coronal Holes and High Speed Wind Streams, ed. J. B. Zirker (Boulder, Col.: Colorado Associated Press)

Withbroe, G. L., \& Noyes, R. W. 1977, ARA\&A, 15,363 